\title{
On the radioactive shells in peculiar main sequence stars: the phenomenon of Przybylski's star.
}

\author{
Vera F. Gopka ${ }^{1}$, Alexander V. Yushchenko ${ }^{2,1}$, Angelina V. Shavrina ${ }^{3}$, \\ David E. Mkrtichian ${ }^{2,4}$, Artie P. Hatzes ${ }^{5}$, Sergey M. Andrievsky ${ }^{4}$ and \\ Larissa V. Chernysheva ${ }^{6}$ \\ ${ }^{1}$ Astronomical observatory, Odessa national University, Park Shevchenko, Odessa, 65014, \\ Ukraine \\ email:gopkavera@mail.ru \\ ${ }^{2}$ Astrophysycal Research Center dor the Structure and Evolution of the Cosmos, Sejong \\ University, Seoul, 143-747, (South) Korea \\ email: yua@arcsec.sejong.ac.kr, david@arcsec.sejong.ac.kr \\ ${ }^{3}$ Main Astronomical observatory of the Academy of Science of Ukraine, Kiev, 03680, Ukraine \\ email: shavrina@mao.kiev.ua \\ ${ }^{4}$ Department of astronomy, Odessa National University, Odessa, 65014, Ukraine \\ email: scan@deneb.odessa.ua \\ ${ }^{5}$ Thuringer Landessternwarte, Tautenburg, D-07778, Germany \\ email: artie@tls-tautenburg.de \\ ${ }^{6}$ A.F. Ioffe Physical-technical institute, Saint-Petrsburgh, 194021, Russia \\ email: Larissa.Chernysheva@mail.ioffe.ru
}

\begin{abstract}
Using high-dispersion spectral observations of HD 101065 (Przybylski's star) we have identified the lines of heavy radioactive elements with atomic numbers from 84 to 99 in the spectrum of the star. We found the lines of all these elements except At $(Z=85)$ and $\operatorname{Fr}(Z=87)$. We try to explain a presence of such heavy species in the atmosphere of this star as a result of radioactive decay of Th and U nuclei and neutron capture process producing some transuranium isotopes. The necessary conditions for these processes to work may appear due to atomic diffusion and favorable accumulation of the thorium and uranium nuclei in the upper of the PS atmosphere.
\end{abstract}

Keywords. Atomic data, nucleosynthesis, stars: abundances, stars: chemically peculiar, stars: individual (HD 101065)

\section{Introduction}

In 1961 Przybylski (1961) discovered a star with properties that placed it far outside of the known limits of the stellar peculiarity. The most outstanding feature of this star is its anomalously high atmospheric abundance of the rare elements like lanthanides. Later, Cowley (2003) and Cowley et al. (2004) identified the lines of radioactive technetium and promethium in spectrum of this star. It should be noted that the presence of the thorium and uranium lines was also reported for this star. The long-standing problem connected to the Przybylski's star (hereafter PS) was its iron abundance. All these peculiarities have no exhaustive explanation up to now.

The abundance pattern of PS now consists of 57 elements. 54 elements were investigated by Cowley et al. (2000), the abundance of lithium was found by Shavrina et al. (2001, 2003), the identification of the lines of Tc and Pm were made by Cowley et al. 
(2004). This is the second stellar abundance pattern (the first is that of the Sun - 73 elements). But the appearance of the spectrum is still a puzzle! There are a lot of strong and faint unidentified lines.

An idea to search for the line of radioactive elements in spectrum of PS (with life-times shorter than billion years) was dominated some works performed in the past century and devoted to this star. For instance, Cowley (2000) reported the remarkable overabundance of the thorium and uranium in PS atmosphere at the level of about 2.5 dex (in the scale $\log N(\mathrm{H})=12$ ). "Can the peculiarities of HD 101065 can be explained by fission of transuranium elements" - this is the title of Przybylski's (1975) paper.

A discussion on the abundance of Pm and Tc in PS and HR465, started from Aller \& Cowley (1970), consists of several dozens papers and summarized by Cowley et al. (2004). We continue such investigations, and this paper presents the first results on the new identification of the lines of radioactive elements in PS's spectrum.

\section{Observations and spectrum synthesis}

For our spectroscopic analysis we have used the high-resolution spectra of PS obtained by one of as $(\mathrm{AH})$ during four observational nights (March, 2004) at 3.6 meter ESO telescope with resolving power near $R=110000$ in the wavelength region 3780$69124 \AA$ (HARPS spectrograph). Reduction and wavelength calibration of these spectra was made by two of us (AH \& DM). VLT spectra (resolving power of about 80000 , $S / N>300$ ) covering the interval from $3040 \AA$ to $10400 \AA$, and the spectra taken at the ESO 1.4 meter CAT in 1996 (resolving power is $100000, S / N>300$, spectral interval 6675-6735 Åand 6120-6180 ̊) were used also.

These spectra were compared to synthetic spectrum generated using the Kurucz's numeric code SYNTHE and PS atmosphere model calculated by Pavlenko (Shavrina et al. 2003) with the following parameters $T_{\text {eff }}=6600 \mathrm{~K}, \log g=4.2$ and additional absorption in lines of rare earth elements. The microturbulent velocity of $2.0 \mathrm{~km} \mathrm{~s}^{-1}$ and projected rotational velocity $v \sin i=3.5 \mathrm{~km} \mathrm{~s}^{-1}$ were adopted. The abundances were taken from Cowley at al. (2000). In our spectrum synthesis we used the most recent compilation of the atomic and molecular data described by Yushchenko et al. (2004). It should be noted that we have used the last version of the list of lanthanides lines from DREAM database (Biemont et al. , 2002).

\section{The line identification}

The position of lines of the radioactive elements under search were simply visualized in synthetic spectrum as vertical markers because there are no any atomic data for these lines except for their wavelengths (Sansonetti et al. 2004), enabling one to calculate their profiles with more or less real intensities. In case there was an obvious coincidence between the position of the line of radioactive element and that of the apparent spectral line, and if there was no any significant contribution from the lines of other canonical elements (with reasonable abundances) to this given line, then we then naturally adopted that this spectral line belongs to the considered radioactive element. We selected only lines, which can be used for abundance analysis later, when oscillator strengths and other atomic data of these lines will be available.

Table 1 lists all the lines identified in this way in the PS spectrum. The columns are the laboratory and observed wavelengths, the identifications, the equivalent widths and identifications from Cowley (2003). As a comment to this Table we have to note that 


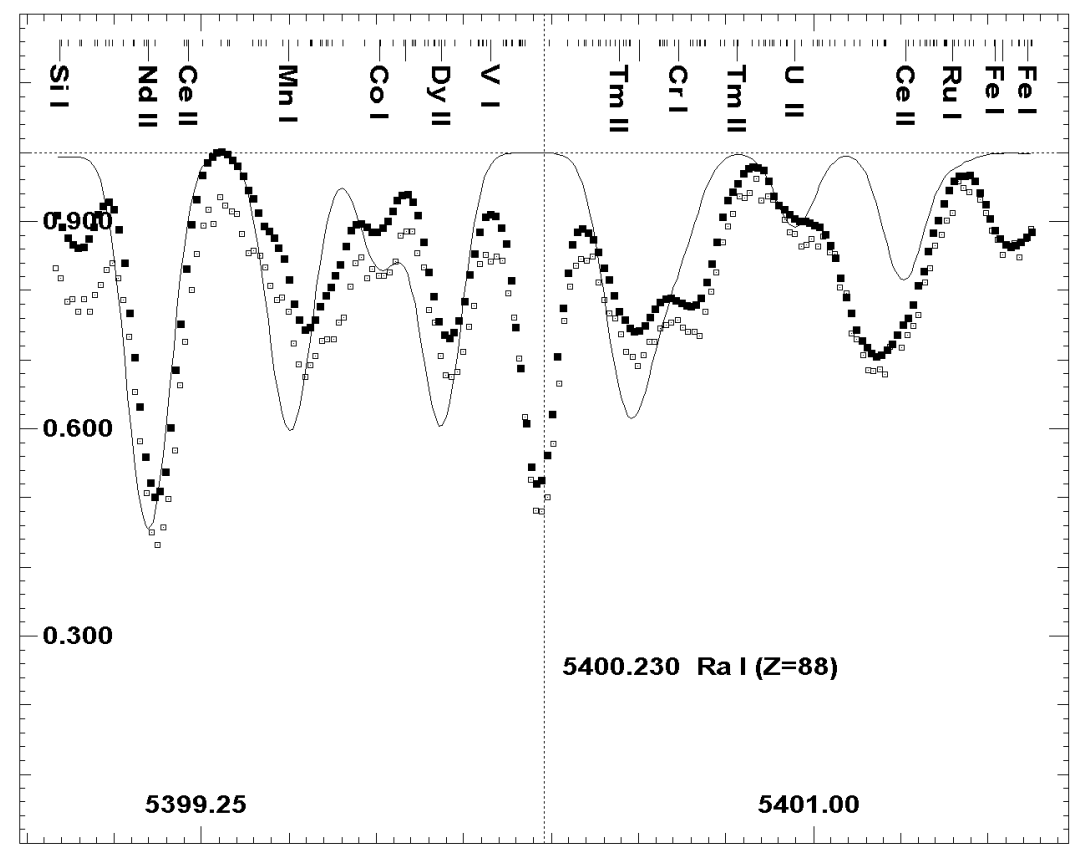

Figure 1. The lines of unstable elements in the spectrum of PS. Filled and open squares are observed spectra, solid lines - synthetic spectra after the convolution with rotational (3.5 $\left.\mathrm{km} \mathrm{s}^{-1}\right)$ and instrumental $(R=110000)$ profiles. Part of the strong lines is marked in the upper part of the figure. The position of radium line is marked by vertical dashed line. The axes are wavelengths in angstroms and relative fluxes.

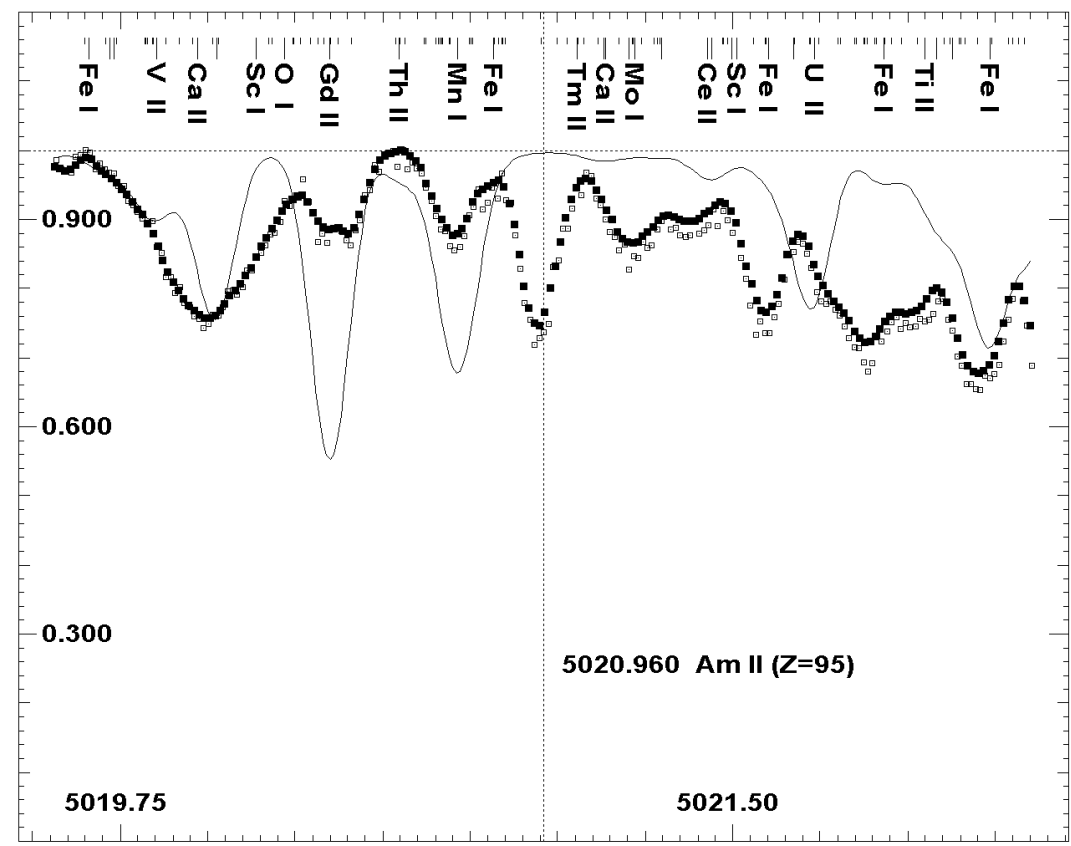

Figure 2. Same as Fig. 1 in the vicinity of line of the Am II $\lambda 5020.960 \AA$. 


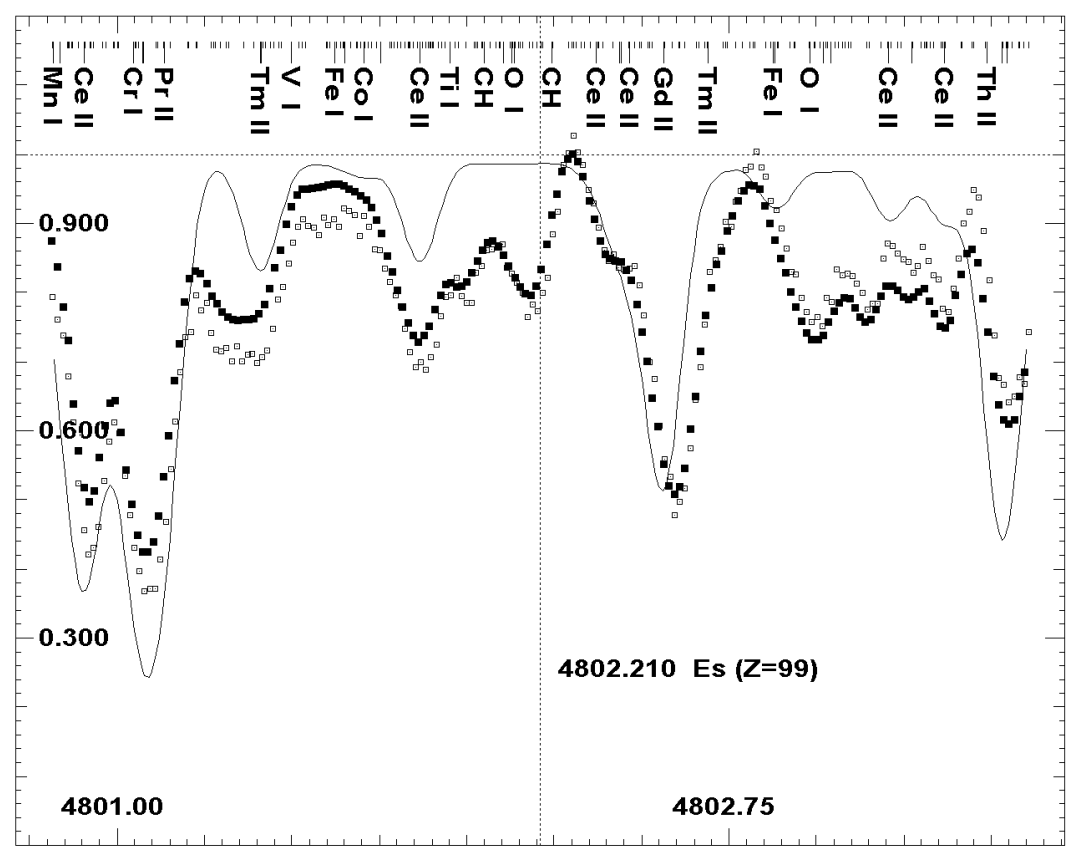

Figure 3. Same as Fig. 1 in the vicinity of line of the Es $\lambda 4802.210 \AA$.

we confirmed previous identifications of the technetium and promethium lines made by Cowley (2004).

Table 1. Lines of radioactive elements in the spectrum of HD 101065

\begin{tabular}{|c|c|c|c|c|c|}
\hline \multicolumn{2}{|l|}{$\lambda$} & \multicolumn{2}{|c|}{ Identification } & \multirow{2}{*}{$\begin{array}{l}\text { Eq. width } \\
(\mathrm{m} \AA)\end{array}$} & Ident. Cowley et al. \\
\hline Lab. & Obs. & Code & Element & & $\begin{array}{ll}\lambda & \text { Ident. }\end{array}$ \\
\hline 3328.600 & .600 & 84.00 & Po I & 13 & 3328.56 no ident. \\
\hline 4508.480 & .475 & 86.00 & Rn I & 38 & \\
\hline 6751.810 & .805 & 86.00 & Rn I & 8 & \\
\hline 8520.950 & .956 & 86.00 & Rn I & 1.6 & \\
\hline 5400.230 & .215 & 88.00 & Ra I & 62 & \\
\hline 7141.210 & .225 & 88.00 & Ra I & 60 & \\
\hline 3043.300 & .298 & 89.01 & Ac II & 25 & \\
\hline 3154.410 & .397 & 89.01 & Ac II & 50 & \\
\hline 3885.560 & .573 & 89.00 & Ac I & in blend & \\
\hline 4194.400 & .397 & 89.01 & Ac II & 72 & 4194.38 no ident. \\
\hline 5758.970 & .964 & 89.01 & Ac II & 22 & 5758.96 no ident. \\
\hline 5910.850 & .866 & 89.01 & Ac II & 24 & 5910.82 no ident. \\
\hline
\end{tabular}


Table 1. Continued

\begin{tabular}{|c|c|c|c|c|c|c|}
\hline \multicolumn{2}{|l|}{$\lambda$} & \multicolumn{2}{|c|}{ Identification } & \multirow{2}{*}{$\begin{array}{l}\text { Eq. width } \\
(\mathrm{m} \AA)\end{array}$} & \multicolumn{2}{|c|}{ Ident. Cowley (2003) } \\
\hline Lab. & Obs. & Code & Element & & $\lambda$ & Ident. \\
\hline 3170.890 & .870 & 91.01 & Pa II & 36: & & \\
\hline 4012.960 & .945 & 91.01 & Pa II & 38: & 4013.00 & .98 ErII \\
\hline 6162.560 & .586 & 91.00 & $\mathrm{PaI}$ & in blend & & \\
\hline 6945.720 & .247 & 91.00 & $\mathrm{PaI}$ & $55:$ & & \\
\hline 7872.950 & .951 & 91.00 & $\mathrm{~Pa} \mathrm{I}$ & 23 & & \\
\hline 6972.090 & .081 & 93.00 & $\mathrm{~Np} I$ & 27 & & \\
\hline 7735.140 & .125 & 93.00 & $\mathrm{~Np}$ I & 7 & & \\
\hline 8529.960 & .948 & 93.00 & $\mathrm{~Np}$ I & & & \\
\hline 9141.300 & .296 & 93.00 & Np I & 41 & & \\
\hline 3220.942 & .936 & 94.01 & $\mathrm{Pu}$ II & 37: & & \\
\hline 3252.070 & .064 & 94.00 & $\mathrm{Pu} \mathrm{I}$ & 47 & & \\
\hline 3320.607 & .593 & 94.00 & $\mathrm{Pu} \mathrm{I}$ & 40 & 3320.61 & no ident. \\
\hline 3792.220 & .255 & 94.00 & $\mathrm{Pu} \mathrm{I}$ & & & \\
\hline 6608.947 & .957 & 94.00 & $\mathrm{Pu} \mathrm{I}$ & 4.5 & & \\
\hline 3446.186 & .194 & 95.00 & Am I & & & \\
\hline 3510.127 & .130 & 95.00 & Am I & & 3510.08 & no ident. \\
\hline 3569.163 & .197 & 95.00 & Am I & 48 & & \\
\hline 3777.504 & .493 & 95.01 & Am II & 75 & 3777.50 & no ident. \\
\hline 4020.252 & .279 & 95.00 & Am I & & 4020.28 & no ident. \\
\hline 4089.291 & .320 & 95.01 & Am II & & 4089.33 & $.41 \mathrm{NbII}$ \\
\hline 4265.550 & .560 & 95.00 & Am I & & 4265.54 & no ident. \\
\hline 4324.570 & .574 & 95.01 & Am II & & 4324.58 & no ident. \\
\hline 5000.210 & .217 & 95.00 & Am I & 97 & 5000.18 & no ident. \\
\hline 5020.960 & .944 & 95.01 & Am II & 34 & 5020.94 & no ident. \\
\hline 5598.130 & .158 & 95.00 & Am I & in blend & 5598.14 & no ident. \\
\hline 6405.110 & .105 & 95.00 & Am I & & 6405.08 & no ident. \\
\hline 3116.411 & .420 & 96.00 & $\mathrm{Cm} \mathrm{I}$ & 32: & & \\
\hline 3147.325 & .333 & 96.00 & $\mathrm{Cm} \mathrm{I}$ & 20: & & \\
\hline 3252.675 & .672 & 96.00 & $\mathrm{Cm} \mathrm{I}$ & & 3252.65 & no ident. \\
\hline 3296.708 & .693 & 96.01 & $\mathrm{Cm} \mathrm{II}+\mathrm{Rh} 96.717$ & & & \\
\hline 3452.922 & .950 & 96.00 & $\mathrm{Cm} \mathrm{I}$ & & 3452.99 & no ident. \\
\hline 4266.450 & .439 & 96.00 & $\mathrm{Cm} \mathrm{I}$ & 17 & 4266.42 & no ident. \\
\hline 4608.400 & .391 & 96.00 & $\mathrm{Cm} \mathrm{I}+\mathrm{CoI} .398$ & & & \\
\hline 6058.900 & .920 & 96.00 & $\mathrm{Cm} \mathrm{I}$ & 14 & 6058.94 & no ident. \\
\hline 6243.350 & .363 & 96.00 & $\mathrm{Cm} \mathrm{I}$ & 88 & 6543.37 & $.33 \mathrm{~atm}$ \\
\hline 6640.170 & .195 & 96.00 & $\mathrm{Cm} \mathrm{I}$ & 4 & & \\
\hline 7720.470 & .477 & 96.00 & $\mathrm{Cm} \mathrm{I}$ & & & \\
\hline 3412.131 & .145 & 97.01 & Bk II & 3 & & \\
\hline 3627.607 & .602 & 97.00 & Bk I & & & \\
\hline
\end{tabular}


Table 1. Continued

\begin{tabular}{|c|c|c|c|c|c|c|}
\hline \multicolumn{2}{|l|}{$\lambda$} & \multicolumn{2}{|c|}{ Identification } & \multirow{2}{*}{$\begin{array}{l}\text { Eq. width } \\
(\mathrm{mA})\end{array}$} & \multicolumn{2}{|c|}{ Ident. Cowley (2003) } \\
\hline Lab. & Obs. & Code & Element & & $\lambda$ & Ident. \\
\hline 3743.047 & .066 & 97.00 & $\mathrm{Bk}$ I & 63 & & \\
\hline 3745.403 & .419 & 97.00 & Bk I & 73 & 3745.42 & no ident. \\
\hline 3802.470 & .480 & 97.00 & $\mathrm{Bk} \mathrm{I}$ & 5 & & \\
\hline 3847.626 & .633 & 97.00 & $\mathrm{Bk} \mathrm{I}$ & 19 & & \\
\hline 3880.106 & .086 & 97.00 & $\mathrm{Bk} \mathrm{I}+.065 \mathrm{~W} \mathrm{I}$ & 25 & & \\
\hline 4147.134 & .135 & 97.01 & Bk II & 39 & 4147.13 . & $12 \operatorname{Pr} I I$ \\
\hline 4423.011 & .017 & 97.00 & Bk I & & 4423.01 & no ident. \\
\hline 5656.540 & .512 & 97.00 & Bk I & 24 & 5656.51 & no ident. \\
\hline 7040.850 & .847 & 97.00 & Bk I & 38 & & \\
\hline 7394.260 & & 97.01 & Bk II & & & \\
\hline 9862.390 & .387 & 97.01 & Bk II & 6 & & \\
\hline 3659.460 & .474 & 98.00 & Cf I & & 3659.46 & no ident. \\
\hline 3699.490 & .472 & 98.01 & Cf II & 67 & 3699.45 & no ident. \\
\hline 4335.220 & .232 & 98.00 & Cf I & 50: & 4335.26 & $.29 \mathrm{GdII}$ \\
\hline 5408.890 & .875 & 98.00 & $\mathrm{Cf}_{\mathrm{I}}$ & & & \\
\hline 3498.110 & .135 & 99.00 & Es I-? & 27 & 3498.10 & no ident. \\
\hline 3575.680 & .691 & 99 & Es & in blend & 35 & \\
\hline 3595.470 & .487 & 99 & Es & 55 & 3595.41 & no ident. \\
\hline 3606.750 & .741 & 99.01 & Es II & 68 & 3606.75 & no ident. \\
\hline 3792.990 & .008 & 99 & Es & 32 & 3792.98 & no ident. \\
\hline 4802.210 & .200 & 99 & Es & 21 & & \\
\hline
\end{tabular}

A small part of the lines, identified by Cowley et al. (2004) appears to be lanthanides lines, but the main result is valid - Tc and Pm exist in the atmosphere of PS. We estimated the abundance of Tc in PS - it appears to be near 3.5-4 in the scale $\log N(\mathrm{H})=12$. Five lines of Tc were used to find the abundance. The abundance of Tc is quite high but of the order of the abundances of the other chemical elements near atomic number $Z=43$ in the atmosphere of PS.

Figures 1-3 show the examples of several lines of the radioactive elements in the spectrum of PS. These are the lines of $\mathrm{Ra}(Z=85), \mathrm{Am}(\mathrm{Z}=95), \mathrm{Es}(\mathrm{Z}=99)$. It should be noted that the information about Es lines in Sansonetti et al. (2004) is extremely limited there is only identification of the element, and no identification of the spectrum (first or second).

\section{Heavy radioactive elements and PS phenomenon}

As one can see from Table 1 the spectrum of PS quite likely contains the lines of heavy radioactive elements with atomic numbers from $Z=84$ to $Z=99$. Then one can ask about how did these heavy unstable elements originate in PS atmosphere? The usual answer is that it is impossible, because of very quick radioactive decay of these elements. To explain the observations we propose the following hypothesis. These heavy nuclides can be created as a result of the radioactive $\alpha$ - and $\beta$-decay of the more long- 
lived isotopes with higher atomic numbers. We should have a stable physical process which can produce additional atoms of unstable isotopes continuously. For example, let us consider two chains of the radioactive decay of Th and Np. The first is ${ }_{90}^{232} \mathrm{Th}$ :
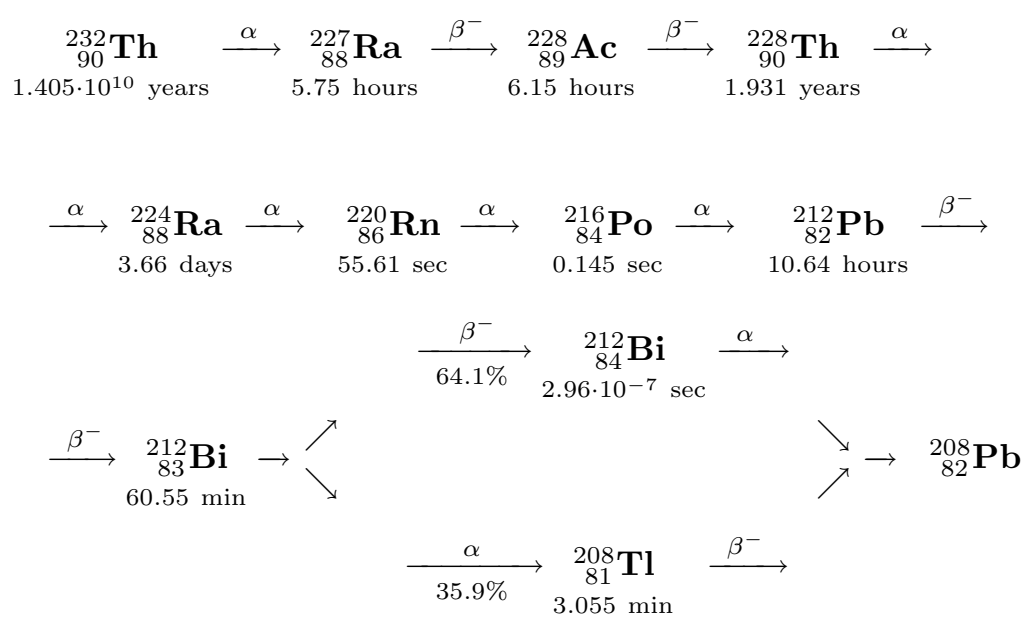

${ }_{93}^{237} \mathrm{~Np}$ decays in the following way:

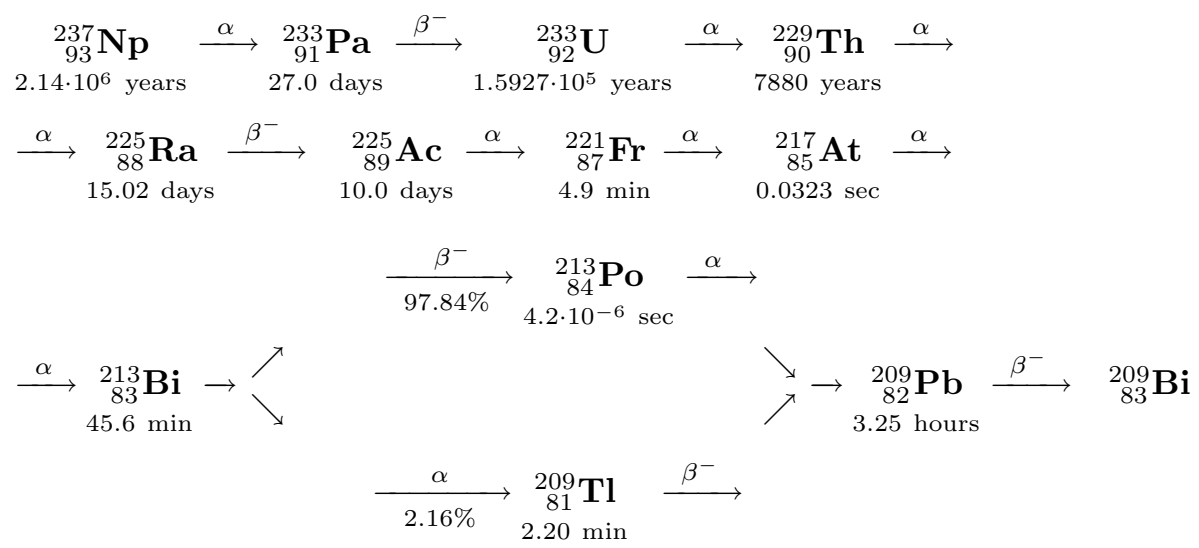

The similar set or reactions can be written for other elements. Proposed mechanism may also quite naturally explain an appearance of the transuranium elements in plasma enriched in thorium and uranium.

Indeed, the radioactive decay of some heavy nuclides is accompanied by emission of $\alpha$-particles and free neutrons. The long-term neutron exposure of uranium and heavier species can lead to creation of some detectable amount of the radioactive isotopes of neptunium ${ }^{237} \mathrm{~Np},{ }^{239} \mathrm{~Np}$, curium ${ }^{244} \mathrm{Cm}$, americium ${ }^{241} \mathrm{Am}$, etc. Such a process is responsible for an appearance of these isotopes in the atomic reactors and in autunites. The macroscopic quantities of elements, heavier than uranium, are produced in industry. These elements exist in nature, so we can expect, that the existence of transuranium elements in stellar atmospheres is possible.

In the PS atmosphere the layers with relatively high concentration of uranium and thorium atoms can be created as a result of atomic diffusion. If undisturbed for the rather long time, these layers may accumulate some amount of radioactive nuclides that originate from the $\mathrm{Th}$ and $\mathrm{U}$ radioactive decay, as well as from neutron capture by thorium and uranium seed nuclei. 
It should be noted that the proposed mechanism means that the abundances of $\mathrm{Pb}$ and $\mathrm{Bi}$ in the atmosphere of the star should be increased, as the abundances of $\mathrm{Th}$ and $\mathrm{U}$ will be decreased with time. The life time of F-type star is too short to notice these variations. We tried to found the upper limits of lead and bismuth abundances in the atmosphere of PS. The limits appeared to be equal 2.8 and 1.4 in the scale $\log N(\mathrm{H})=12$ for $\mathrm{Pb}$ and $\mathrm{Bi}$ respectively. This is of the order of 1 dex higher than the solar abundances of these elements.

Maybe, after calculations of oscillator strengths, part of our identifications will be rejected, first of all first spectra of actinides. Part of the lines can appear to be the lanthanides lines. The number of lanthanides lines with known oscillator strengths is still unsufficient.

It is well known that the lanthanides are overabundant in PS by 4-5 dex. Shavrina et al. (2003) tried to find new lanthanides lines in the vicinity of $\mathrm{Li} 6708 \AA$ line using NIST energy levels. We are going to make similar investigation for all lines of unstable elements. Preliminary results show that the lines of unstable elements can not be explained as a lanthanides lines.

\section{Conclusions}

Having analyzed the high-resolution spectra of PS we have identified the lines of heavy radioactive elements with atomic numbers from $\mathrm{Z}=84$ to $\mathrm{Z}=99$. We identified the lines of all elements except At $(Z=85)$ and $\operatorname{Fr}(Z=87)$. We explain a presence of such heavy species in PS atmosphere as a result of radioactive decay of Th and $U$ nuclei and neutron capture process producing some transuranium isotopes. The necessary conditions for these processes to work may appear due to atomic diffusion and favorable accumulation of the thorium and uranium nuclei in the upper of the PS atmosphere.

In any case it is necessary to check the possibility of proposed mechanism in PS. As was mentioned by Cowley (2003), PS is extreme, but a member of a reasonably well understood class (roAp stars). So the explanation of the peculiarities of this star, HR465 and HD965 should be found in the frames of common physics for this class stars. We should take in mind that astrophysical sites for $r$-process are still debated in the literature. Nucleosynthesis of heavy elements is an open question both in theory and in experiment (see Woosley \& Hoffman 1992 and Adamenko \& Vysotskii 2004 and references therein as an examples).

The additional mechanism to produce abundance anomalies in PS can be the spallation reactions. Shavrina et al. (2003) proposed that these reactions can be responsible for the anomalous ratio of lithium isotopes in the atmosphere of PS. Cowley et al. (2004) pointed that spallation reactions can produce Tc and Pm in PS.

It should be noted that the another investigation of PS, using wavelength coincidence statistics, was performed by Bidelman (2004). He found the list of radioactive elements in $\mathrm{PS}$ - all elements in the range of atomic numbers from $\mathrm{Z}=84$ to $\mathrm{Z}=89$, except $\mathrm{Z}=85$ (At) and $\mathrm{Z}=87$ (Fr) in excellent agreement with our results.

\section{Acknowledgements}

We used data from NASA ADS, SIMBAD, CADC, VALD, NIST, and DREAM databases, UVES Paranal Observatory Project (ESO DDT Program ID 266.D-5655) and we thank the teams and administrations of these projects.

Work by AY and DM was supported by the Astrophysical Research Center for the Structure and Evolution of the Cosmos (ARCSEC) of Korea Science and Engineering Foundation (KOSEF) through the Science Research Center (SRC) program. Work by 
VG was partially supported by research funds of Chonbuk National University, (South) Korea.

\section{References}

Adamenko, S.V., Vysotskii V.I., 2004, http://proton21.org.ua

Aller, M., \& Cowley, C.R. 1970, ApJ 162, 145

Bidelman, W.P. 2004, Private communication

Biemont, J., Palmeri, P., \& Quinet, P. 2002, Database of rare earths at Mons University http://www.umh.ac.be/ astro/dream.html

Cowley, C. 2003, http://astro.1sa.umich.edu/users/cowley/

Cowley, C., Ryabchikova, T., Kupka, F., Bord, D.J., Mathys, G., \& Bidelman, W.P. 2000, MNRAS 317, 299

Cowley, C.R., Bidelman, W.P., Hurbig, S., Matis, G., \& Bord, D.J. 2004, A\&A 419, 1087

Przybylski, A. 1961, Nature 189, 4766, 739

Przybylski, A. 1975, PASAu, Annual General Meeting, 9th, Parker, New South Wales, Australia, May 14-16, 1975 Astronomical Society of Australia, Proceedings. 1975. 2, 352

Sansonetti, J.E., Martin, W.C., \& Young, S.L. 2004., Handbook of Basic Atomic Spectroscopic Data (version 1.1), http://physics.nist.gov/Phys.Ref.Data/Handbook

Shavrina, A.V., Khalack, V., Polosukhina, N.S, Zverko, J., Zhiznovsky, J., Gopka, V.F., North, P., Tsymbal, V.V., \& Yushchenko, A.V. 2001, Odessa Astron. Publ. 14, 249

Shavrina, A.V., Polosukhina, N.S., Pavlenko, Ya.V., Yushchenko, A.V., Quinet, P., Hack, M., North, P., Gopka, V.F., Źverko, J., Žižňovský, J., \& Veles, A. 2003, A\&A 400, 707

Woosley, S.E., Hoffman, R.D. 1992, ApJ, 395, 202

Yushchenko, A., Gopka, V., Kim, C., Liang, Y.C., Musaev, F., \& Galazutdinov, G. 2004, A\&A 413,1105 